\title{
A Low-Voltage Low-Power Fully-Integratable Front-End for Hearing Instruments
}

\author{
Wouter A. Serdijn, Albert C. van der Woerd, Jan Davidse, and Arthur H. M. van Roermund
}

\begin{abstract}
In this paper, the front-end of a universally applicable analog integrated circuit for hearing instruments is presented. This IC comprises the following functions: a microphone preamplifier, a pickup-coil preamplifier, a second-order high-pass filter and a second-order low-pass filter, both with a controllable cutoff frequency, and an input-controlled automatic gain control with an adjustable knee level. By applying a compression/expansion system and operating all the circuits in the current domain as much as possible, all these functions can be implemented in a single IC, without the need for external components. The test chip demonstrates operation down to $1.05 \mathrm{~V}$ and a current consumption between 120 and $175 \mu \mathrm{A}$. The full-custom chip area in a $2.5-\mu \mathrm{m}$ BiCMOS process (using only vertical NPN's and lateral PNP's) amounts to $2.4 \mathrm{~mm}^{2}$.
\end{abstract}

\section{INTŔODUCTION}

$\mathbf{M}$ ORE AND MORE people have great difficulty in understanding speech in surroundings with background noise and/or reverberation. It is estimated that in the industrialized countries about $10 \%$ of all people suffer from hearing impairment [1]. Among them is a large number of elderly people; $42 \%$ is older than 65. Apart from these people, also people on the work floor run the risk of becoming subject to severe hearing damage: $45 \%$ of the hearing impaired is between 25 and 45 years old. Younger people may become hard of hearing, as a direct result of listening to their favorite music or playing in their own band. And as if all that is not enough, also the ears of babies and little children are in danger from noisy toys,

All these people may become easily isolated by the lack of communication with other people in their immediate surroundings. This is particularly true in situations such as informal get-togethers, parties, meetings, cafés, and playgrounds.

Hearing impairment may be due to various causes, either external or pathological, but is frequently the result of the aging of the auditory organ itself [2]. This often entails both the attenuation and the distortion of sounds [3]. It is the distortion of sounds especially that results in a worse speech intelligibility in noisy surroundings.

In the past, various devices have been invented to assist those with impaired hearing. Until 1900, they were often shaped as a tapered horn. In the horn, the sound energy at the large opening is concentrated towards a small opening on the side of the ear. After the turn of the century, the first com-

Manuscript received January 31, 1995; revised July 12, 1995. This paper was recommended by Guest Editors A. Rodríguez-Vázquez and E. SánchezSinencio.

The authors are with the Delft University of Technology, Faculty of Electrical Engineering, $2628 \mathrm{CD}$ Delft, The Netherlands.

IEEE Log. Number 9415056. mercially available electrical hearing aids were introduced [4]. Initially, they only consisted of a microphone, a telephone, and a bulky battery. Amplification was achieved in the transduction process from acoustic to electrical signals in the carbonmicrophone. These early hearing aids were of poor quality and provided limited gain. A major turning point came with the invention of electron tubes, the first active components, and the development of practical electronic amplifiers [5].

When the transistor was invented, smaller apparatus could be developed. At the end of the fifties, the first hearing instruments of the behind-the-ear type appeared. These had an enormous advantage over all the earlier types: They could be worn almost invisibly. Some types were also supplied with a pick-up coil for listening via an induction-loop system, as used in, e.g., churches, theaters, and other public buildings.

In the eighties, hearing instruments that could be worn inside the ear appeared. Apart from the cosmetic effect, their main advantages are the small size, and the natural placement of the microphone, which enables directional hearing. With the progress in IC technology other features became possible. Today there are remote-controlled, digitally programmable, and self-adapting apparatus.

It must be noted that amplification alone is often insufficient, because not only the desired sound but also the noise is amplified. In this case, some improvement of the speech intelligibility can be obtained by applying signal processing in the frequency or time domain [6]. For this reason, many types of today's hearing instruments are equipped with filters, gain controls (whether automatic or not), limiters or a compression/expansion circuitry [7].

Since 1990, our project group "Low-Voltage Low-Power Electronics" of the Electronics Research Laboratory has been engaged in the research and development of universally applicable analog integrated circuits for hearing instruments. This project is being carried out in close cooperation with an industrial partner. The functions to be incorporated are

- a microphone preamplifier

- a pickup-coil preamplifier

- a high-pass filter with adjustable cutoff frequency

- a low-pass filter with adjustable cutoff frequency

- an input-controlled automatic gain control (AGC-I) with adjustable knee level

- an output-controlled automatic gain control (AGC-O) with adjustable knee level

- a volume control

- a maximum-gain control

- a gain-tolerance control 
- a power amplifier

- a $-30 \mathrm{~dB}$ output, used for driving external power amplifiers in so-called super-power hearing aids

- a microphone supply

The organization of the paper is as follows. After description of the system specifications in Section II, the system setup is discussed in Section III. The front-end of the system consists of the following elementary building blocks: Three filters, a controlled microphone preamplifier, an envelope processor, an expander and a pickup-coil preamplifier. These are treated in Sections IV-VIII. The other parts (forming the rear-end of a hearing instrument) are not discussed in this paper. The semicustom realization and its measurement results are the subject of Section IX. After a brief glance at a full custom realization in Section X, the conclusions are given in Section XI.

\section{SPECIFICATIONS}

The integrated circuitry of the front-end had to fulfill the following requirements:

\section{A. General Parameters}

- chip area: preferably less than $5 \mathrm{~mm}^{2}$

- supply voltage: $1.1-1.6 \mathrm{~V}$

- supply impedance: $0-100 \Omega$

- current consumption: preferably less than $300 \mu \mathrm{A}$

- temperature range: $10-45^{\circ} \mathrm{C}$

- preferably no external components

\section{B. Audio Parameters}

1. Output signal: The interface between the front-end and the rear-end of the hearing instrument is a signal current of maximally $5 \mu \mathrm{A}$ (peak value).

\section{Bandwidth and distortion:}

- bandwidth: at least from $150 \mathrm{~Hz}$ to $8 \mathrm{kHz}(-3 \mathrm{~dB})$

- distortion: $<7 \%$ when input signal is between 14 and 28 $\mathrm{mV}$ rms and $<2 \%$ when input signal is less than $14 \mathrm{mV}_{\mathrm{rms}}$

3. Microphone preamplifier:

- source impedance: $4.4 \mathrm{k} \Omega$ in series with $33 \mathrm{nF}$

- input impedance: $50 \mathrm{k} \Omega$

- input referred noise of total hearing instrument:

a) $\max 2 \mu \mathrm{V}_{\mathrm{rms}}$ A-weighted when gain is between max. gain and $26 \mathrm{~dB}$ below max gain

b) $10 \mu \mathrm{V}_{\text {rms }} \mathrm{A}$-weighted when gain is $40 \mathrm{~dB}$ below max gain is tolerable

- max. input signal:

a) $28 \mathrm{mV}_{\mathrm{rms}}$ unweighted for frequencies higher than $50 \mathrm{~Hz}$.

b) $56 \mathrm{mV}_{\mathrm{rms}}$ unweighted for frequencies lower than $50 \mathrm{~Hz}$.

\section{Pickup-coil preamplifier:}

- transimpedance amplifier, ${ }^{1}$ gain adjustable from 6-60 k $\Omega$ with an external resistor

${ }^{1}$ Although the output voltage of the pickup coil is proportional to the magnetic flux, the linearity of the transfer is not essentially different when using either voltage or current sensing. In the latter case, however, the transfer also depends on the coil impedance. Together with a coupling capacitor this leads to a second-order low-frequency roll-off, which can improve the speech intelligibility. For this reason current sensing is used here.
- load is the input impedance of the microphone preamplifier

- max. output voltage: $24 \mathrm{mV}_{\mathrm{rms}}$

- source impedance: $L$ in series with $R, L$ between 500 and $900 \mathrm{mH}, \mathrm{R}=2 \mathrm{k} \Omega$

- output impedance: about $4 \mathrm{k} \Omega$, in correspondence with the microphone impedance

- output noise: $<10 \mu \mathrm{V}_{\mathrm{rms}}$ flat between $100 \mathrm{~Hz}$ and $10 \mathrm{kHz}$

- max. current consumption: $<30 \mu \mathrm{A}$

5. High-Pass filter:

- order: 2

- cutoff frequency: 100-1600 Hz (linearly adjustable in octaves)

- stop-band attenuation: $>40 \mathrm{~dB}$

6. Low-Pass filter:

- order: 2

- cutoff frequency: $1.6-8 \mathrm{kHz}$ (linearly adjustable in octaves)

- stop-band attenuation: $>30 \mathrm{~dB}$

7. AGC-I:

- attack time: $<5 \mathrm{~ms}$

- release time: $80 \mathrm{~ms}$

- knee level: $60 \mu \mathrm{V}_{\mathrm{rms}}-28 \mathrm{mV}_{\mathrm{rms}}$ (linearly adjustable in dB's)

- compression ratio above knee level: 2

\section{A COMPRESSION/EXPANSION SYSTEM}

From the input-referred noise and the maximum input signal, it follows that the dynamic range of the total hearing instrument must be at least $28 \mathrm{mV} / 2 \mu \mathrm{V}=83 \mathrm{~dB}$. This requires of all the circuits in the signal path that their dynamic range is at least more than this $83 \mathrm{~dB}$. Due to the limited supply voltage, this is a problem especially in circuits that have a voltage as the information-carrying quantity somewhere inside, such as filters [8]. We thus can expect that the design of filters with more than $83-\mathrm{dB}$ dynamic range is the hardest part of the total design.

In [9], a controllable second-order high-pass filter has been presented that, with some minor modifications, could be applicable in this design. However, for a 83-dB dynamic range, instead of $50 \mathrm{~dB}$, this would require a total capacitance of $36 \mathrm{nF}$, which is unacceptable in a fully-integrated realization. For the above reasons, a compression/expansion solution has been chosen. The key idea is that, in order to fulfill the dynamic range requirements of the total circuit, the signal must be compressed before it is passed to the "noisy" filters. Afterwards, the signal is expanded again to restore the original input-output relation. Thus, the noise is always masked by the signal, leading to a virtually higher dynamic range. As the total circuit already contains a compressor (AGC-I) only one additional expander is needed. The operation of the total compression/expansion system is as follows. The compressor compresses the input signal when it becomes larger than a fixed reference level. This reference level corresponds with the lowest knee level of the AGC-I ( $\left.60 \mu \mathrm{V}_{\mathrm{rms}}\right)$. In the compressor also a control signal is generated that contains information 


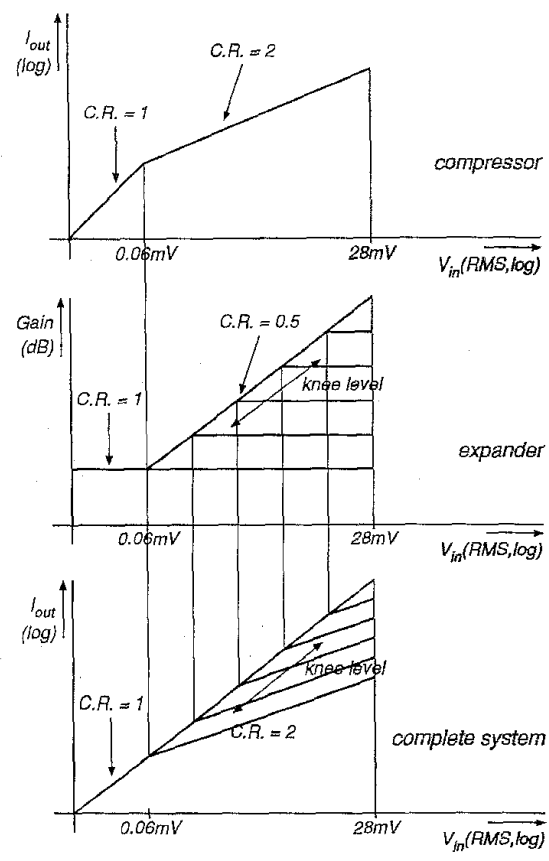

Fig. 1. Transfer as function of the input signal of the compressor, the expander, and the complete system.

about the magnitude of the input signal. This signal controls the gain of the controlled amplifier in the compressor but can also be used to control the gain of the expander. In the expander the control signal is compared with the knee level. If the input signal is smaller than the knee level the expander will expand; the input-output relation becomes linear again. If the input signal is larger than the knee level the expander must no longer be controlled by the control signal; the input-output relation remains compressed. Note that in this system the knee level thus is set by the expander and not by the compressor. See Fig. 1.

Another point of concern is offset. In bipolar integrated circuits offset occurs as a product of base currents and mismatch between the various components. For this reason the secondorder high-pass-filter function is realized by means of two first-order high-pass filters in cascade. These two filters can be inserted at places where the offset may become critical and thus they act as offset filters. Of course, when used in this way, the poles of the high-pass filter function cannot be complex. However, this is no problem in this application.

As discussed in [11], a compressor consists of three parts: a controlled amplifier, a comparator and an integrator. Instead of using a microphone preamplifier with a fixed transfer followed by a controlled amplifier, it is also possible to provide the microphone preamplifier with a controlled output. This reduces the complexity of the total circuit. The remaining circuitry of the compressor, viz. the comparator and the integrator, are called from now on the envelope processor.

During the design process, it also appeared that too much offset could be expected to originate from the microphone preamplifier. For the high-pass filter this does not pose a

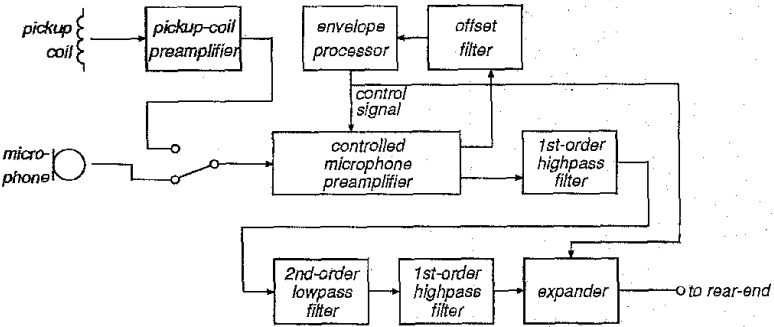

Fig. 2. Block diagram of the front-end.

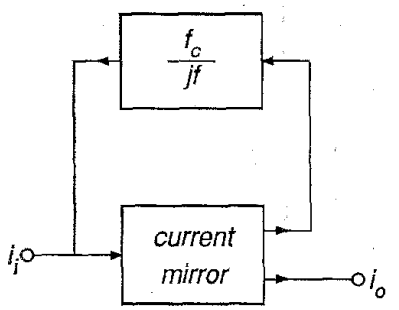

Fig. 3. A first-order high-pass leap frog filter operating in the current domain.

problem as it acts as an offset filter itself. However, for the envelope processor, the offset leads to uncertainty in the value of the knee level. Therefore an additional offset filter has been placed between the microphone preamplifier and the envelope processor.

The resulting block diagram is depicted in Fig. 2. As this is a typical low-voltage low-power analog integrated circuit, current has been chosen as the information-carrying quantity wherever possible [12]. The interface between the various circuits is thus performed by currents.

\section{THE FILTERS}

As has been mentioned earlier, it is to be expected that the filters set an upper limit to the dynamic range of the total hearing instrument. We therefore discuss them first.

\section{A. The Two First-Order High-Pass Filters}

The starting point of our discussion is a first-order highpass leapfrog filter operating in the current domain [9], as depicted in Fig. 3. The filter consists of an integrator (because of its frequency stability) and a current mirror with multiple outputs. The input-output relation $H(f)$ is given by

$$
H(f)=\frac{i_{o}}{i_{i}}=\frac{-1}{1+f_{c} / j f}
$$

in which $f_{c}$ equals the cutoff frequency of the filter.

For the integrator we have taken a capacitancetransconductance amplifier with enlarged voltage swing [8]. See Fig. 4. This integrator realizes the transfer function

$$
H_{X}=\frac{n}{j 2 \pi f R_{1}\left(1-A_{V}\right) C}
$$

with $A_{V}=-R_{2} / R_{1}$. 


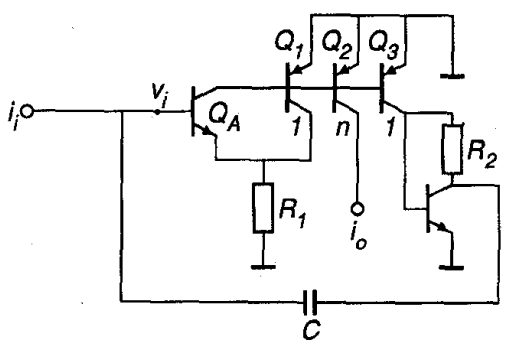

Fig. 4. Signal path of the integrator.

The scaling factor $n$ sets the cutoff frequency $f_{c}$ of the filter. Combining (1) and (2), we find that $n$ must satisfy

$$
n=2 \pi f_{c} R_{1}\left(1-A_{V}\right) C
$$

The noise performance of the integrator can be optimized by varying the collector current of the first stage $Q_{A}$ resulting in an optimal collector current

$$
I_{C, Q_{A}, \mathrm{opt}}=\frac{V_{T}}{\sqrt{R_{1}^{2} / B_{F}+1 / 4 \pi^{2} B_{F}\left(1-A_{V}\right)^{2} C^{2} f_{1} f_{2}}}
$$

$f_{1}$ and $f_{2}$ being the lowest and highest frequency of the audio bandwidth, respectively.

The maximum output current $i_{o, \max }$ of the integrator can be determined from the maximum input voltage $V_{i, \max }$. With (3), it follows that

$$
i_{o, \max }=V_{i, \max } n / R_{1}=V_{i, \max } 2 \pi f_{c, \min }\left(1-A_{V}\right) C
$$

$f_{c, \text { min }}$ being the minimal cutoff frequency of the filter.

The tuning of the filter is done by varying the factor $n$ which equals the collector current of $Q_{2}, I_{C, Q_{2}}$ divided by the collector current of $Q_{1}, I_{C, Q_{1}}$. With (3), it follows:

$$
I_{C, Q_{1}}=I_{C, Q_{2}} / n=I_{C, Q_{2}} / 2 \pi f_{C} R_{1}\left(1-A_{V}\right) C .
$$

Only a proper choice for the resistors $R_{1}$ and $R_{2}$ remains. Therefore, we look at the efficiency of the integrator. In view of the power efficiency $n$ is best chosen larger than one for all cutoff frequencies possible. With (3), it follows that

$$
n=2 \pi f_{c} R_{1}\left(1-A_{V}\right) C>1 \quad \forall f_{c}
$$

and thus

$$
R_{1}>\frac{1}{2 \pi f_{c, \min }\left(1-A_{V}\right) C}
$$

With $f_{c, \min }=100 \mathrm{~Hz}, C=400 \mathrm{pF}$ and $A_{V}=-5$ this results in: $R_{1}>660 \mathrm{k} \Omega$ and $R_{2}=-A_{V} R_{1}>3.3 \mathrm{M} \Omega$. These are very large values for an IC. Apart from this the noise behavior is seriously degraded by such a large $R_{1}$. A compromise therefore has been adopted: $R_{1}=40 \mathrm{k} \Omega$ and $R_{2}=200 \mathrm{k} \Omega$.

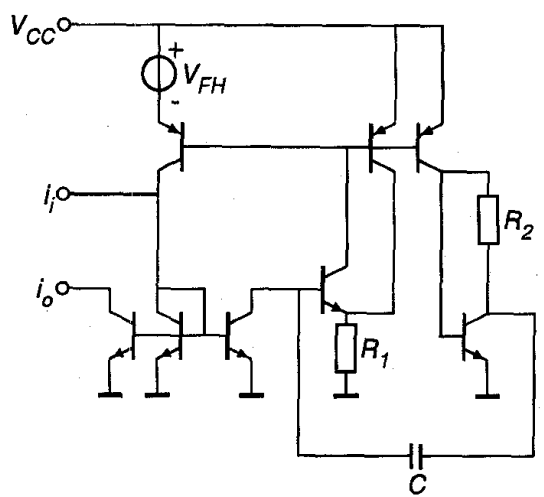

Fig. 5. Circuit diagram of the high-pass filter.



Fig. 6. The high-pass filter including its biasing scheme.

When we combine the integrator with a current mirror according to the block diagram shown in Fig. 3, we have completed the signal path of the filter. See Fig. 5. The adjustable scaling factor $n$, which determines the cutoff frequency of the filter, is obtained by means of an adjustable voltage source $V_{F H}$

$$
n=e^{-V_{F H} / V_{T}} .
$$

With (3), it follows:

$$
f_{c}=\frac{n}{2 \pi R_{1}\left(1-A_{V}\right) C}=\frac{e^{-V_{F H} / V_{T}}}{2 \pi R_{1}\left(1-A_{V}\right) C} .
$$

This exponential relation between $V_{F H}$ and the cutoff frequency means that when $V_{F H}$ varies linearly the cutoff frequency varies in octaves. If $V_{F H}$ is made proportional to the absolute temperature (PTAT) the cutoff frequency also becomes independent of the temperature.

The complete filter, including its biasing scheme, is depicted in Fig. 6.

All the necessary bias currents are delivered by one PTAT current source with multiple outputs. The voltage $V_{F H}$ originates from another circuit. This circuit is not discussed here. Transistors $Q_{B}$ through $Q_{H}$ provide the collector current of 


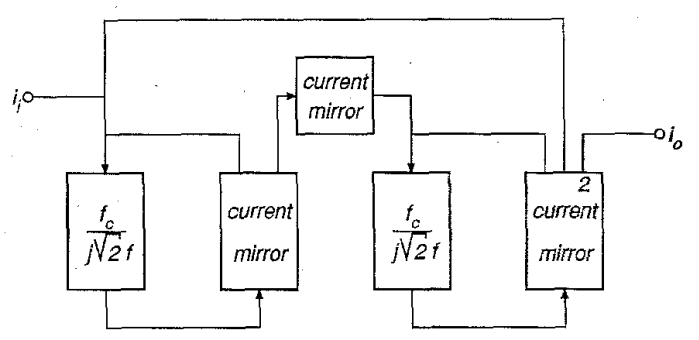

Fig. 7. A second-order low-pass Butterworth leapfrog filter operating in the current domain.

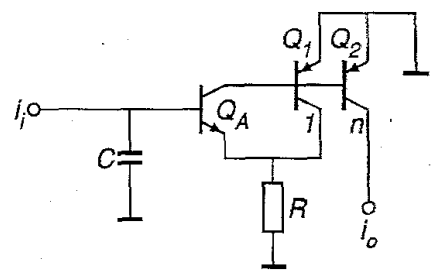

Fig. 8. Circuit diagram of the integrator.

$Q_{3}$. The terminals $V_{P}, V_{N}$ and $V_{T}$ all are connected with the PTAT current source and are used for the biasing of the other circuits. Capacitor $C_{X}$ increases the phase margin of the shunt stage to avoid instability.

\section{B. The Second-Order Low-Pass Filter}

The starting point is the second-order low-pass leapfrog filter as depicted in Fig. 7. This filter operates in the current domain, consists of two integrators, two current mirrors with multiple outputs and an ordinary current mitror. The third output of the right mirror equals twice the (inverted) input, to obtain a zero-loss passband transfer. The input-output relation $H_{f}$ of the filter is given by

$$
H_{f}=\frac{i_{o}}{i_{i}}=\frac{-1}{j^{2} f^{2} / f_{c}^{2}+j \sqrt{2} f / f_{c}+1}
$$

in which $f_{c}$ equals the cutoff frequency of the filter. Note that for every $f_{c}$ the filter response is maximally flat (i.e., a Butterworth characteristic).

For the integrator, we have taken a capacitancetransconductance amplifier [9]. See Fig. 8. This integrator realizes the transfer function

$$
H_{I}=\frac{n}{j 2 \pi f R C} .
$$

The scaling factor $n$ sets the cutoff frequency $f_{c}$ of the filter. Combining (11) and (12), we find that $n$ must satisfy

$$
n=\pi \sqrt{2} f_{c} R C \text {. }
$$

The noise performance of the integrator can be optimized by varying the collector current of the first stage $Q_{A}$ yielding an optimal collector current

$$
I_{C, Q_{A}, \mathrm{opt}}=\frac{V_{T}}{\sqrt{R^{2} / B_{F}+1 / 4 \pi^{2} B_{F} C^{2} f_{1} f_{2}}} .
$$

The maximum output current $i_{o, \max }$ of the integrator can be determined from the maximum input voltage, $V_{C, \max }$. With (13), it follows that

$$
i_{o, \max }=v_{C, \max } n / R=v_{C, \max } \pi \sqrt{2} f_{c, \min } C
$$

$f_{c, \text { min }}$ being the minimal cutoff frequency of the filter.

The tuning of the filter is done by varying the factor $n$. With (13), it follows

$$
I_{C, Q_{1}}=I_{C, Q_{2}} / n=I_{C, Q_{2}} / \pi \sqrt{2} f_{c} R C
$$

Only a proper choice for the resistor $R$ remains. For that, we look at the efficiency of the integrator. Again, $n$ is best chosen to be larger than one for all possible cutoff frequencies. With (13), it follows that

$$
n=\pi \sqrt{2} f_{c} R C>1 \quad \forall f_{c}
$$

and thus

$$
R>\frac{1}{\pi \sqrt{2} f_{c, \min } C} .
$$

With $f_{c, \text { min }}=100 \mathrm{~Hz}$ and $C=100 \mathrm{pF}$ this results in $R>1.4 \mathrm{M} \Omega$. This is a very large value for an IC. Apart from this, the noise behavior is seriously degraded by such a large value. A compromise therefore has been adopted: $R=140 \mathrm{k} \Omega$.

When we combine the two integrators with three current mirrors according to the block diagram shown in Fig. 7, we have completed the signal path of the filter. See Fig. 9. The adjustable scaling factor $n$, which determines the cutoff frequency of the filter, is obtained by means of an adjustable voltage source $V_{F L}$

$$
n=e^{-V_{F L} / V_{T}}
$$

With (13), it follows:

$$
f_{C}=\frac{n}{\pi \sqrt{2} R C}=\frac{e^{-V_{F L} / V_{T}}}{\pi \sqrt{2} R C} .
$$

This exponential relation between $V_{F L}$ and the cutoff frequency means that when $V_{F L}$ varies linearly the cutoff frequency varies in octaves. If $V_{F L}$ is made proportional to the absolute temperature (PTAT) the cutoff frequency also becomes independent of the temperature.

The complete filter, including its biasing scheme, is depicted in Fig. 10.

All the necessary currents are delivered by the PTAT current source with multiple outputs in the high-pass filter (terminal $V_{P}$ ). The voltage $V_{F L}$ originates from another circuit. This circuit is not discussed here. Transistors $Q_{B}$ through $Q_{I}$ provide the collector currents of $Q_{1 A}$ and $Q_{1 B}$.

\section{The Offset Filter}

The starting point for the design of the offset filter is the first-order high-pass leapfrog filter operating in the current 


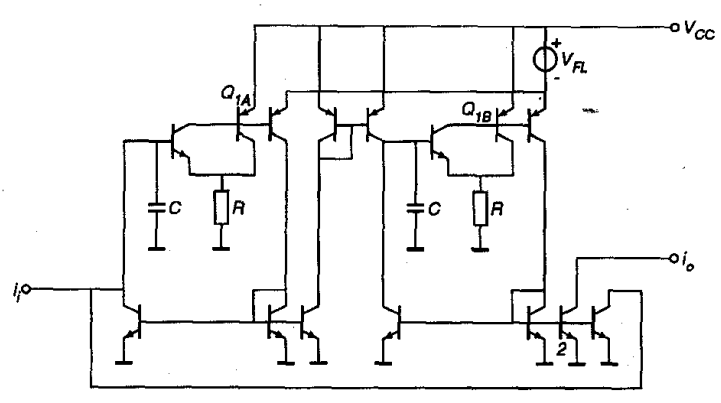

Fig. 9. Signal path of the low-pass filter.

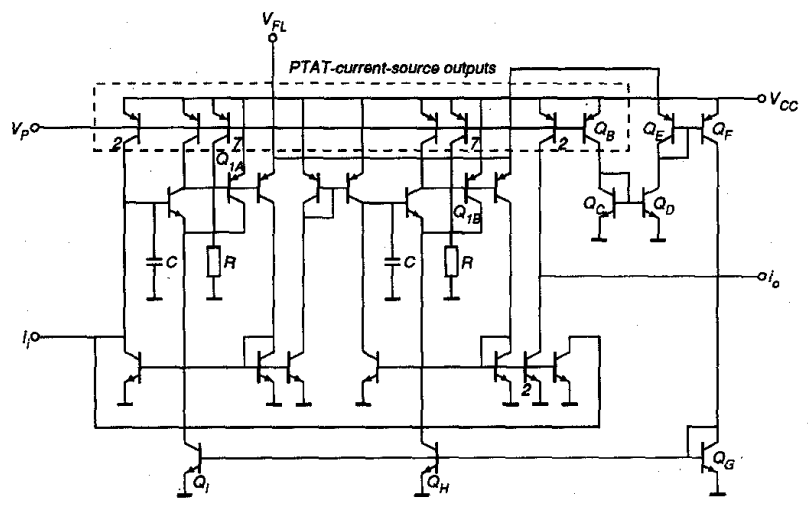

Fig. 10. The low-pass filter including its biasing scheme.

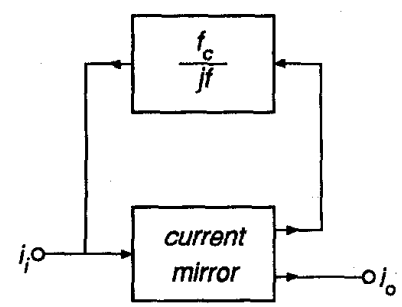

Fig. 11. A first-order high-pass leapfrog filter operating in the current domain.

domain according to Fig. 3. See Fig. 11. The filter consists of an integrator (because of its frequency stability) and a current mirror with multiple outputs. The input-output relation $H(f)$ is given by

$$
H(f)=\frac{i_{o}}{i_{i}}=\frac{-1}{1+f_{c} / j f}
$$

in which $f_{c}$ equals the cutoff frequency of the filter.

In order to keep the voltage across the (small) integrating capacitor in the integrator within bounds the filter is scaled with a scaling factor $\alpha$ [10]. This gives the filter shown in Fig. 12. The current mirror provides two different outputs. One output equals the inverted input. The other equals $1 / \alpha$ times the inverted input. This output is fed back to the integrator. Note that the transfer functions of both filters are the same.

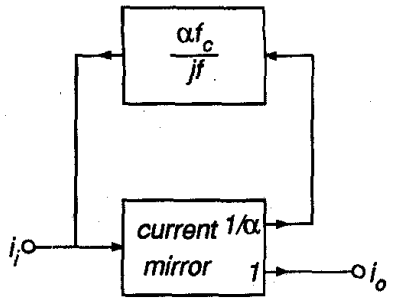

Fig. 12. A scaled first-order high-pass leapfrog filter operating in the current domain.

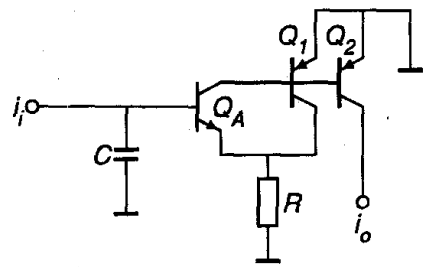

Fig. 13. Circuit diagram of the integrator.

For the integrator, we have taken the circuit depicted in Fig. 13. This integrator realizes the transfer function

$$
H_{I}=\frac{1}{j 2 \pi f R C} .
$$

Combining (21) and (22), we find that $\alpha$ must satisfy

$$
\alpha=\frac{1}{2 \pi f_{c} R C} \text {. }
$$

As the offset filter is not part of the signal path of the total front-end, its noise performance is of minor importance. For this reason, the collector current of the first stage of the integrator is not optimized with respect to the noise contribution of the integrator, but with respect to its influence on the offset current at the output of the total filter. $I_{C, Q_{A}}$ therefore simply equals the sum of the base currents of $Q_{1}$ and $Q_{2}$.

The maximum output current $i_{o, \max }$ of the integrator can be determined from the maximum input voltage, $V_{C, \max }$. With (22), it follows that

$$
i_{o, \max }=V_{C, \max } / R
$$

This determines the value of $R$.

When we combine the integrator with a current mirror according to the block diagram shown in Fig. 12, we have completed the signal path of the filter. See Fig. 14. The scaling factor $1 / \alpha$ is obtained by means of a fixed voltage source $V_{X}$ in series with the emitter of the scaled transistor

$$
\alpha=e^{V_{X} / V_{T}} .
$$

With (23), it follows:

$$
f_{c}=\frac{1}{2 \pi \alpha R C}=\frac{e^{-V_{X} / V_{T}}}{2 \pi R C} .
$$

If $V_{X}$ is proportional to the absolute temperature (PTAT) the cutoff frequency also is independent of the temperature. 


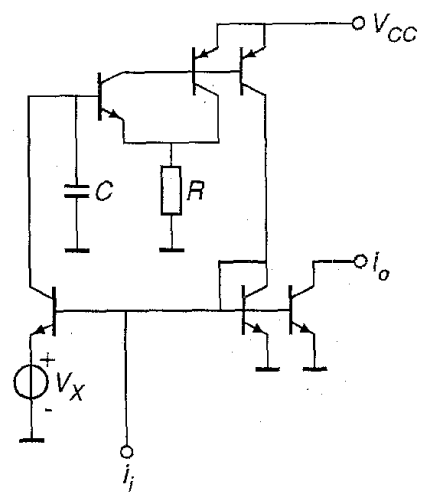

Fig. 14. Signal path of the offset filter.

The complete filter, including its biasing scheme, is depicted in Fig. 15.

To reduce the offset current at the output of the filter, the correct values of the collector currents of $Q_{1}$ and $Q_{2}$ are provided by a dummy version of the integrator and transistors $Q_{B}$ through $Q_{E}$. The operation is as follows. The output current of the dummy integrator is compared with a bias current coming from the PTAT current source with multiple outputs (terminal $V_{P}$ ). The error signal is amplified by $Q_{B}$ through $Q_{E}$ and fed back to the input of the dummy integrator. As the absolute value of the loop gain is much larger than one, the error signal is nullified; the dummy integrator is biased correctly. As the collector current of $Q_{D}$ equals the collector current of $Q_{E}$ and the integrators are identical (for dc), the integrator also is biased correctly.

The voltage source $V_{X}$ is realized by means of a resistor $R_{3}$ through which flows a current. This current also originates from the PTAT current source (terminal $V_{T}$ ). The capacitor $C_{X}$ prevents instability of the dummy integrator.

As the absolute value of the offset-filter output signal is to be compared with a reference level-this is done in the envelope processor-the output signal is fed through a rectifier before it is fed to the envelope processor.

\section{THE CONTROLled Microphone PREAMPLIFIER}

The next step in the design of the hearing-instrument frontend is the design of the controlled microphone preamplifier. As this circuit is the first in the total signal path, its noise performance is of major importance. It deserves, therefore, special attention.

There are three possibilities of realizing an amplifier with a 50-k $\Omega$ input impedance. We can choose a current amplifier with a $50-\mathrm{k} \Omega$ resistor in series with its input terminals. Although this is an easy solution, it is not applicable here, because the noise contributed by this resistor would be too large. Another solution might be a transconductance amplifier with a $50-\mathrm{k} \Omega$ resistor in parallel with its input terminals. However, this would turn out to be in conflict with a low current consumption. Probably the best solution is realizing the input impedance by means of a two-loop negative feedback amplifier [13], [14]. We have chosen for this solution. As the



Fig. 15. The offset filter including its biasing scheme.

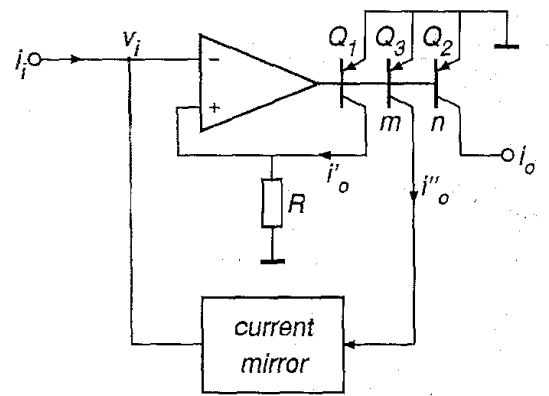

Fig. 16. Basic configuration of an amplifier with a fixed input resistance and a current output.

input of the first high-pass filter and of the offset filter is a current, we thus need an amplifier with a fixed input resistance and a current output. The basic configuration of this amplifier is depicted in Fig. 16.

This type of amplifier can be considered as consisting of a combination of a transconductance amplifier and a current amplifier. The operation is as follows. The input voltage $v_{i}$ is transformed by resistor $R$, the op amp and transistor $Q_{1}$ into a current $i_{o}^{\prime}=v_{i} / R$. $Q_{2}$ provides the output current $i_{o}=n v_{i} / R . Q_{3}$ provides another indirect output current $i_{o}^{\prime \prime}=m v_{i} / R$, which is inverted by the current mirror and fed back to the input. As $i_{o}^{\prime \prime}=i_{i}$ we find for the input impedance $R_{i}$ of the power-to-current converter

$$
R_{i}=v_{i} / i_{i}=R / m
$$

For the transfer function $H_{t}$ of the preamplifier, we can write

$$
H_{t}=i_{o} / v_{i}=n / R \text {. }
$$

From (27) and (28), we see that $R_{i}$ and $H_{t}$ can be chosen independently by means of the scaling factors $m$ and $n$.

Even if the op amp is noise-free the amplifier adds some noise to the signal. This noise contribution originates from the resistor $R$ and transistors $Q_{1}, Q_{2}$ and $Q_{3}$. Their noise sources can be shifted towards the input and summed in one noise voltage source $v_{n, \text { eq }}$, with a power density spectrum $S\left(v_{n, e q}\right)$, 


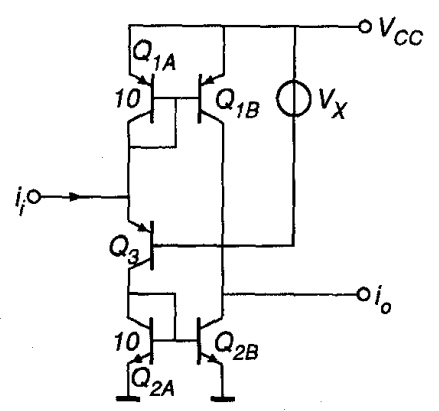

Fig. 17. A simple class-B scaling current mirror.

in series with the signal source. We obtain

$$
\begin{aligned}
S\left(v_{n, \mathrm{eq}}\right)= & 4 k T R+R^{2}\left(2 q I_{C, Q_{1}}+\frac{2 q I_{C, Q_{2}}}{n^{2}}\right) \\
& +2 q I_{C, Q_{3}}\left|Z_{S}\right|^{2}
\end{aligned}
$$

$Z_{S}$ being the source impedance (in this situation the 4.4-k $\Omega$ microphone resistance and the $33-\mathrm{nF}$ coupling capacitor).

With $I_{C, Q_{1}}=I_{C, Q_{2}} / n=I_{C, Q_{3}} / m$ this can be rewritten as

$$
S\left(v_{n, \mathrm{eq}}\right)=4 k T R+2 q I_{C, Q_{1}}\left(R^{2}+\frac{R^{2}}{n}+\frac{\left|Z_{S}\right|^{2}}{m}\right) .
$$

From this expression, we see that $R$ is best chosen to be as small as possible. However, if $R<1 / H_{t}, I_{C, Q_{2}}$ becomes too large. This degrades the power efficiency. A compromise therefore has been adopted: $R=5 \mathrm{k} \Omega$. For a $50-\mathrm{k} \Omega$ input impedance the scaling factor $m$ thus must equal 0.1 . Unfortunately, this increases the noise again. The problem has been overcome by realizing the scaling factor $m$ not in the power-to-current amplifier but in the current mirror. The resulting scaling current mirror is realized in class-B. When there is little signal at the input its noise production is also small. A simple class-B scaling current mirror is depicted in Fig. 17.

This class-B mirror consists of two ordinary current mirrors $\left(Q_{1 A}\right.$ and $Q_{1 B}, Q_{2 A}$ and $\left.Q_{2 B}\right)$ and a CB stage $\left(Q_{3}\right)$. If the incoming current $i_{i}$ is negative, all the current flows into the upper mirror; the CB stage and the lower mirror are off. If $i_{i}$ is positive, the current flows via the CB stage to the lower mirror; the upper mirror is off. At the output, both the positive and negative half of the input signal are added and the original signal is restored.

We now return to the design of the total preamplifier.

To here, the op amp has been considered to be noise-free. In practice, an op amp always contributes some noise. A possible implementation of the op amp is a single CE stage. The circuit diagram of the preamplifier becomes with this $\mathrm{CE}$ stage as depicted in Fig. 18.

We can shift the two noise sources of the CE stage towards the input of the amplifier and transform them into one equivalent noise voltage source $v_{n, \text { eq }}$ in series with the input terminals. In [8], it is found that the power density spectrum of this noise source equals

$$
S\left(v_{n, \mathrm{eq}}\right)=S\left(v_{n}\right)+S\left(i_{n}\right)\left|Z_{S}+R\right|^{2} .
$$

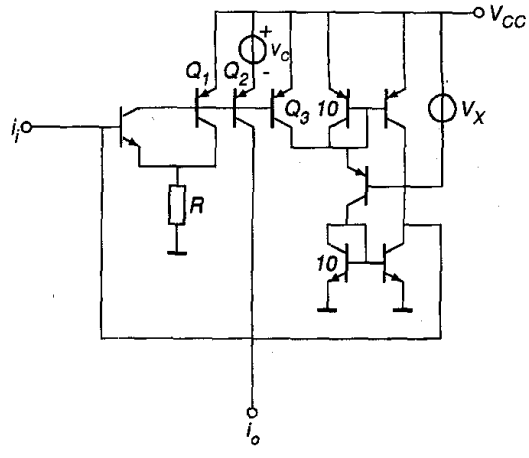

Fig. 18. Circuit diagram of the preamplifier.

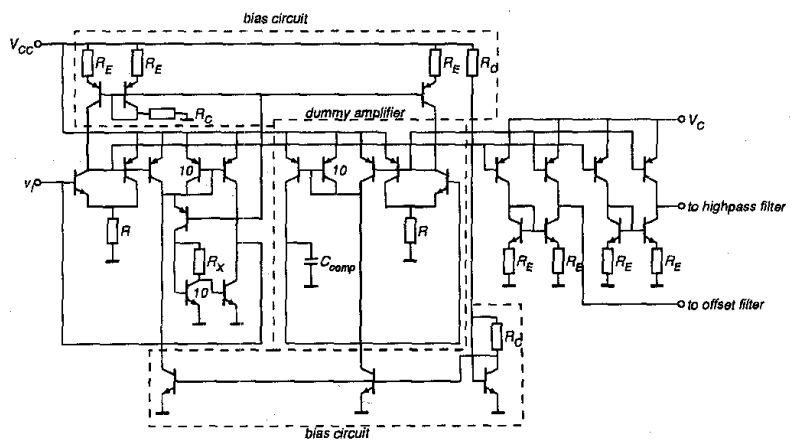

Fig. 19. The controllable microphone preamplifier including its biasing scheme.

Following the same procedure as in the preceding section, an optimal collector current for the $\mathrm{CE}$ stage $I_{C, \mathrm{CE} \text {,opt }}$ can be found

$$
I_{C, \mathrm{CE}, \mathrm{opt}}=\frac{V_{T}}{\sqrt{\left(\bar{R}+R_{S}\right)^{2} / B_{F}+1 / 4 \pi^{2} B_{F} C_{S}^{2} f_{1} f_{2}}}
$$

$R_{S}$ and $C_{S}$ being the microphone resistance and the coupling capacitor value, respectively. With $R_{S}=4.4 \mathrm{k} \Omega, C_{S}=$ $33 \mathrm{nF}, B_{F}=100$ and $R=5 \mathrm{k} \Omega$ this optimal current equals $28 \mu \mathrm{A}$. However, $5 \mu \mathrm{A}$ is also acceptable in this design. This only slightly deteriorates the noise behavior of the preamplifier. In view of the power efficiency $5 \mu \mathrm{A}$ is even better.

The transfer of the preamplifier is made controllable by the scaling factor $n$. This scaling factor is realized by means of a controllable voltage source $V_{C}$

$$
n=e^{-V_{C} / V_{T}} \text {. }
$$

With (28), it follows:

$$
H_{t}=i_{o} / v_{i}=n / R=\frac{e^{-V_{C} / V_{T}}}{R} .
$$

The complete preamplifier, including its biasing circuitry, is depicted in Fing. 19.

As the preamplifier must have two controlled outputs-one to be connected with the first high-pass filter, the other with the offset filter-an extra output has been added. To reduce offset at both outputs, the amplifier is made almost symmetrical by 
means of a dummy amplifier. The collector bias currents are delivered either by the current mirror with two outputs (above) or by the gm-compensated current mirror (below). The emitter resistors $R_{E}$ are used to improve the noise behavior of the total preamplifier.

The resistor $R_{X}$ in the class-B current mirror reduces the current consumption at high input levels. When the input signal is large, there is a large voltage drop over $R_{X}$ and the scaling factor of the NPN part of the class-B current mirror decreases-the NPN mirror is, in fact, a gm-compensated mirror. Therefore, the input current, and thus the current consumption, decreases.

The capacitor $C_{\text {comp }}$ prevents the microphone preamplifier from becoming unstable.

\section{THE ENVELOPE PROCESSOR}

Together, the controlled microphone preamplifier and the envelope processor perform the AGC-I function. In [11], it is shown that we have three possibilities of realizing an automatic gain control with a finite compression ratio. All three contain two controlled amplifiers. However, in the AGC variant with a controlled knee level-the third one--the demands that are made upon the controlled amplifier that controls the knee level can be much fewer. This reduces the circuit complexity and for this reason we have chosen this variant. See Fig. 20.

The operation is as follows. The rectified output signal $E_{o}^{\prime}$ is compared with a reference level $E_{K}^{\prime}$. If $E_{o}^{\prime}<E_{K}^{\prime}$ the integrator is charged by quantity $E_{\text {rel }} ; E_{\text {int }}$ increases, resulting in a larger output signal. If $E_{o}^{\prime}>E_{K}^{\prime}$ the integrator is discharged by $E_{\text {att }}-E_{\text {rel }}\left(E_{\text {att }}>E_{\text {rel }} !\right) ; E_{\text {int }}$ decreases, resulting in a smaller output signal. If $m<0$, variations of the input signal thus always result in smaller variations of the output signal. In [11] we found that the compression ratio-the ratio of the variation of the input signal and the variation of the output signal, both in dBs-equals $1-m$. For a compression ratio of two, $m$ thus equals -1 ; the divider becomes an inverter. With (34), we find for the transfer function $H_{m}$ of the controlled amplifier

$$
H_{m}=\frac{I_{K}^{\prime}}{I_{K}}=e^{-E_{\mathrm{int}}}=e^{V_{C} / V_{T}} .
$$

The current-domain implementation of the envelope processor is depicted in Fig. 21. Apart from $E_{\text {int }}$ all the quantities are represented by currents. The voltage follower generates a low-impedance version of the voltage across the capacitor $C$, to avoid interaction.

For the release time $t_{\text {rel }}$ and the attack time $t_{\mathrm{att}}$, it is found that

$$
t_{\mathrm{rel}}=\frac{2.6 V_{T} C}{I_{\mathrm{rel}}}
$$

and

$$
t_{\text {att }}=\frac{2.6 V_{T} C}{I_{\text {att }}-I_{\mathrm{rel}}}
$$

Fig. 22 shows the circuit diagram of the envelope processor with ideal bias sources.

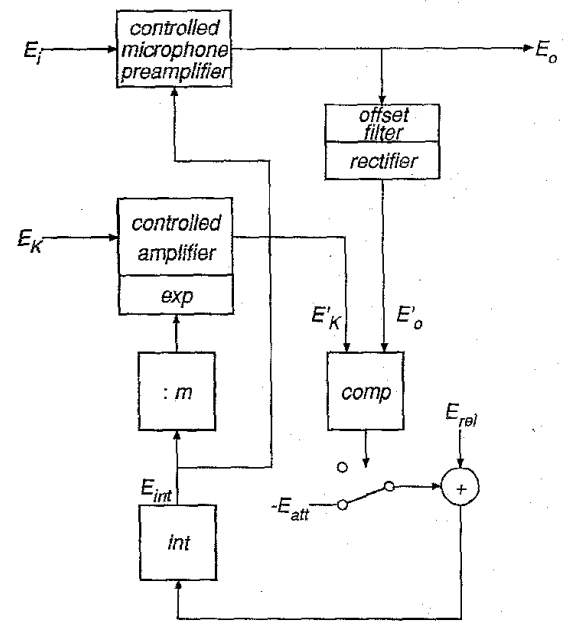

Fig. 20. General block diagram of an AGC with C.R. $=1-m$ using a controlied knee level.

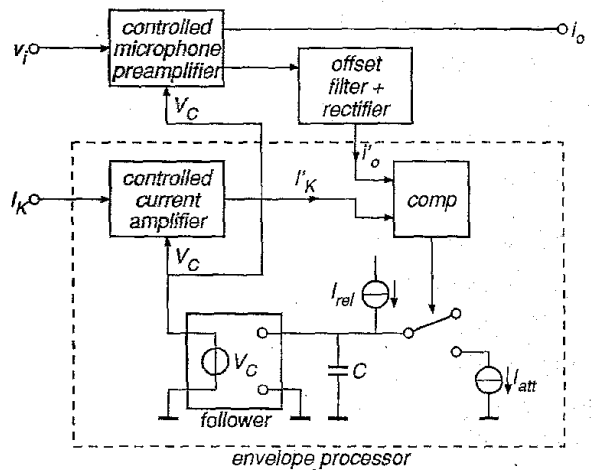

Fig. 21. Current domain implementation of the envelope processor.

The controlled current amplifier has been realized by means of a scaling current mirror. The output current of the controlled microphone preamplifier is subtracted from $I_{K}^{\prime}$. If the remainder is negative, $Q_{S}$ becomes nonconducting and the capacitor $C$ is discharged by the current $I_{\text {att }}-I_{\text {rel }}$. If the remainder is positive, $Q_{S}$ saturates and $C$ is charged by $I_{\text {rel }}$. The voltage follower is realized by means of transistors $Q_{A}$ through $Q_{D} \cdot I_{1}, I_{2}$ and $I_{3}$ provide the collector currents of these transistors.

The reference current $I_{K}$ corresponds with the lowest knee level of the AGC-I $(60 \mu \mathrm{V}$. The actual knee level is set by the expander.

Fig. 23 shows the circuit diagram of the total envelope processor including its bias circuitry. $R_{C}$ realizes the current $I_{3}$. All the other currents are derived from a gm-compensated current mirror with multiple outputs. The input current of this mirror originates from the PTAT current source (terminal $\left.V_{N}\right)$.

\section{THE EXPANDER}

In Section MI, we discussed the function of the expander, which is to expand the signal as long as the input signal of the microphone preamplifier is beneath the knee level of the AGC-I function; if the transfer function of the expander is pro- 


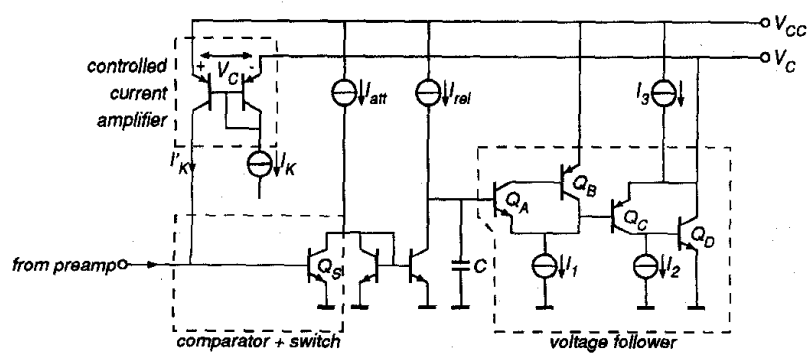

Fig. 22. The envelope processor with ideal bias sources.

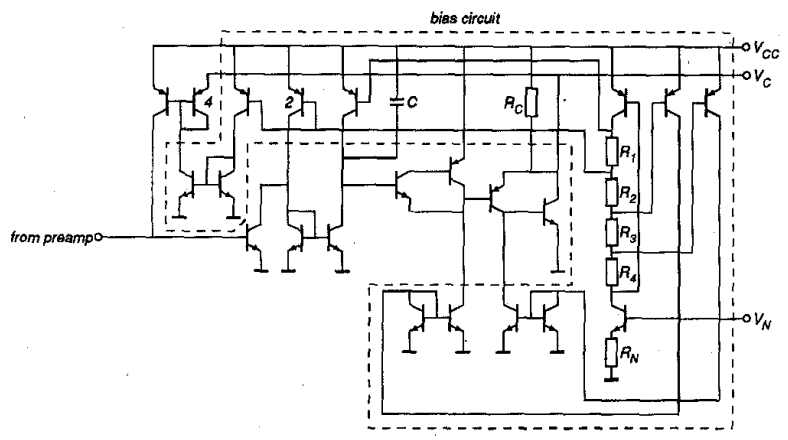

Fig. 23. Circuit diagram of the envelope processor including its bias circuitry.

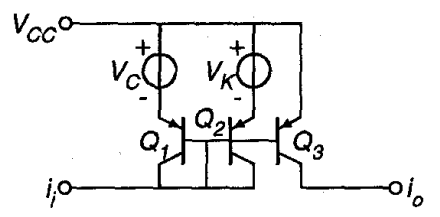

Fig. 24. Circuit diagram of the expander.

portional to the inverse function of the controlled microphone preamplifier, then the total input-output relation becomes linear again. However, if the input signal is above the knee level the transfer of the expander is linear and the total system functions as a compressor with a compression factor of 2 .

This can be accomplished by a simple circuit, which is depicted in Fig. 24.

Its operation is as follows. If $V_{C}<V_{K}$ the base-emitter voltage of $Q_{2}$ is smaller than the base-emitter voltage of $Q_{1}$, and the transfer is determined by $Q_{1}, Q_{3}$ and $V_{C}$ only. In formula

$$
H_{e}=e^{V_{C} / V_{T}}, V_{C}<V_{K} .
$$

If $V_{C}>V_{K}$ the base-emitter voltage of $Q_{2}$ is larger than the base-emitter voltage of $Q_{1}$, the transfer is determined by $Q_{2}, Q_{3}$ and $V_{K}$ only, or

$$
H_{e}=e^{V_{K} / V_{T}}, V_{C}>V_{K} .
$$

If $V_{C}$ is the same control voltage that is generated in the envelope processor and that is used to control the microphone preamplifier, the input-output relation $H_{\text {front-end }}$ of the total front-end satisfies

$$
H_{\text {front-end }}=H_{t} H_{e}=\left\{\begin{array}{cc}
1 / R, & V_{C}<V_{K} \\
e^{V_{K}-V_{C} / V_{T}} / R, & V_{C}>V_{K}
\end{array}\right.
$$

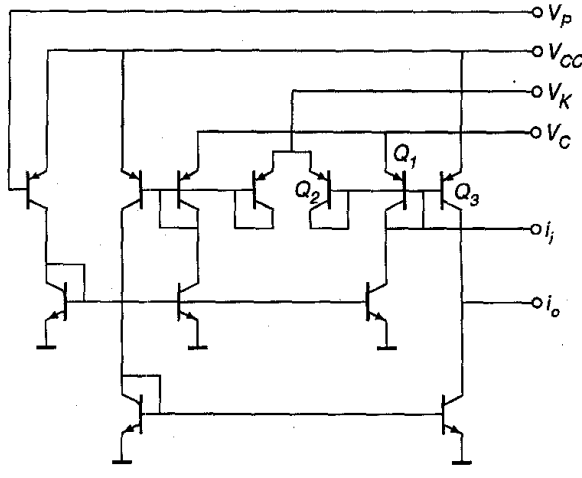

Fig. 25. The expander including its bias circuitry.

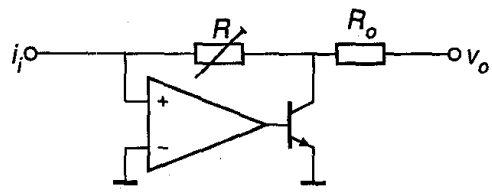

Fig. 26. Basic configuration of the transimpedance amplifier.

It must be noted that the filters need not to be taken into account since they all possess a 0 -dB transfer in the passband. The exponential relation between $V_{K}$ and the knee level means that when $V_{K}$ varies linearly, the knee level varies in dB's. If $V_{K}$ is made proportional to the absolute temperature (PTAT), the knee level also becomes independent of the temperature.

The circuit diagram of the expander including its bias circuitry is depicted in Fig. 25. In order to reduce the offset current at the output, a dummy version of the collector current of $Q_{3}$ is generated and subtracted from this collector current. The other collector currents are derived from the PTAT current source (terminal $V_{P}$ ). The voltage source $V_{K}$ originates from another circuit. This circuit is not discussed here.

\section{THE PICKUP-COIL PREAMPLIFIER}

Finally the design of the pickup-coil preamplifier: A transimpedance amplifier with a gain which is to be adjustable from $6-60 \mathrm{k} \Omega$ by an external resistor. Its output is connected to a switch which passes either this signal-in the $T$ position-or the microphone signal-in the $M$ position-to the input of the microphone preamplifier. For this reason, the output impedance of the pickup-coil preamplifier must approximately equal the microphone impedance $(4 \mathrm{k} \Omega)$.

The basic configuration of a transimpedance amplifier [13] is depicted in Fig. 26. The additional resistor $R_{O}$ has been inserted to obtain the desired output impedance.

For the ideal transfer $A_{f, \infty}$ we can write

$$
A_{f, \infty}=-R \text {. }
$$

A possible implementation of the op amp might be a short circuit. This results in a shunt stage. However, the loop gain of this one-transistor transimpedance amplifier is too small, which causes inaccuracy, distortion or a poor power efficiency. We thus need more amplifying stages in the signal path. A 


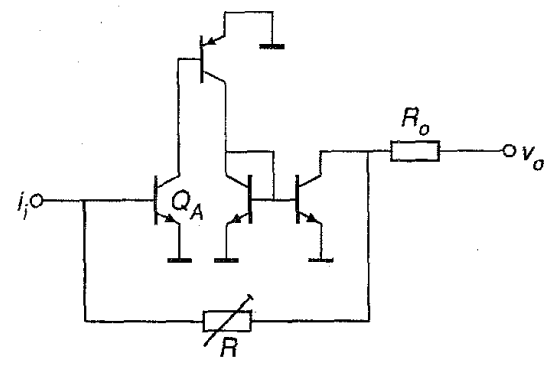

Fig. 27. A four-transistor transimpedance amplifier.

practical solution is given in Fig. 27. The circuit consists of two CE stages in cascade and a current mirror, the low-voltage counterpart of a $\mathrm{CB}$ stage [8]. The noise behavior of this amplifier is determined mainly by the noise coming from the first stage $Q_{A}$ and the resistor $R$. With regard to the noise contribution of the first stage, an optimal collector current can be found, which, unfortunately, becomes too large with regard to the power efficiency. A compromise therefore has been adopted: $10 \mu \mathrm{A}$.

The collector current of the three other transistors depends on the output capability of the amplifier. $10 \mu \mathrm{A}$ is a very convenient value.

Analyzing the high-frequency behavior of this anplifier we find that the closed loop contains three dominant poles, which cause the circuit to oscillate. However, this problem can easily be overcome by adding one additional transistor connected between input and output. See Fig. 28. We now obtain a multipath structure [15]. Its operation is as follows. At low frequencies the loop gain is delivered by the parallel connection of the four-transistor amplifier and the multipath transistor and, as the gain of the four-transistor amplifier is much larger, thus mainly determined by this one. At higher frequencies the multipath transistor takes over, because its dominant pole lies at a much higher frequency than the dominant poles of the four-transistor amplifier. Therefore, the phase margin can be as high as $90^{\circ}$. The capacitor $C_{X}$ has been inserted to ensure that the dominant poles of the fourtransistor amplifier indeed lie at a much lower frequency than the dominant pole of the single CE stage.

The pickup-coil preamplifier including its bias circuitry is depicted in Fig. 29.

All collector currents are derived from a current mirror with two outputs. The emitter resistors $R_{E}$ reduce the noise contribution of this mirror.

\section{SEMICUStom REALIZATION OF THE FRONT-END}

All the circuits presented in the foregoing sections have been integrated in two semicustom chips fabricated at the Delft Institute of Microelectronics and Submicrontechnology (DIMES). Experiment results proved the correct operation of the two high-pass filters, the low-pass filter, the microphone preamplifier, the envelope processor, the expander and the pickup-coil preamplifier. Only the offset filter did not function properly, due to a layout error. In order to test the total frontend, a breadboard version of the offset filter was realized, according to the same circuit diagram and using transistor

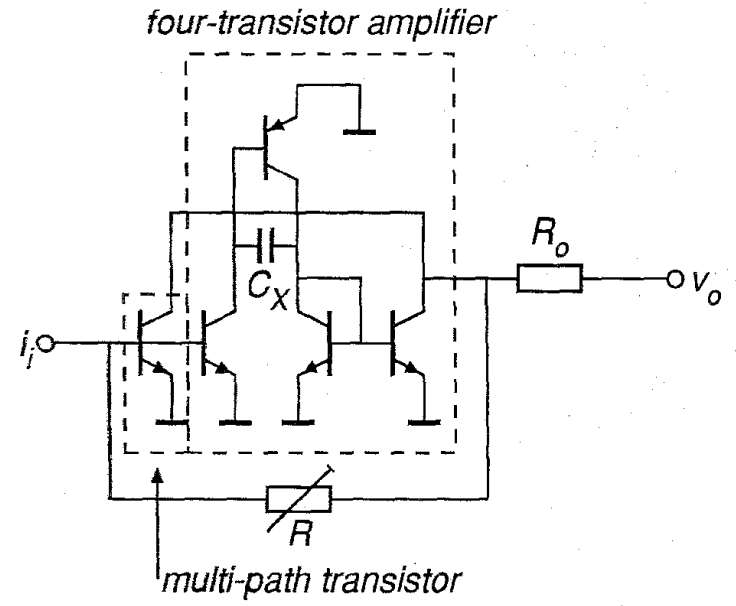

Fig. 28. Circuit diagram of the pickup-coil preamplifier.

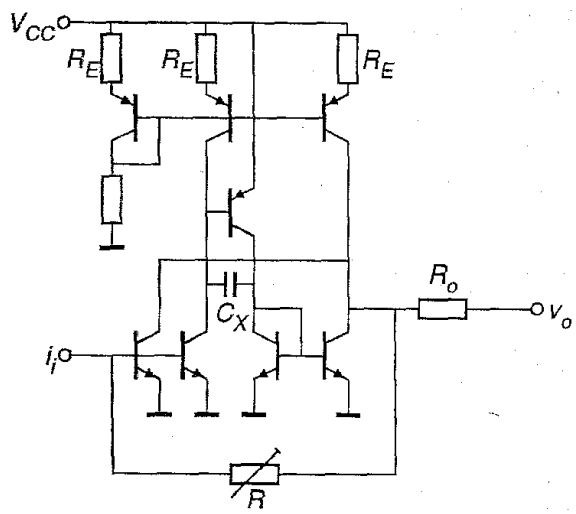

Fig. 29. The pickup-coil preamplifier including its bias circuitry.

arrays fabricated in the same process. This offset filter operated correctly. The measurement results are discussed in the following subsections.

\section{A. General Parameters}

- supply voltage $\left(35^{\circ} \mathrm{C}\right): 1.05-1.9 \mathrm{~V}$

- current consumption $\left(35^{\circ} \mathrm{C}\right): 120-175 \mu \mathrm{A}$

- temperature range $(1.3 \mathrm{~V}):-40-+100^{\circ} \mathrm{C}$

\section{B. Audio Parameters}

1. Input-output transfer:

- $i_{L} / v_{S}=100 \mu \mathrm{A} / \mathrm{V}$

2. Bandwidth and distortion:

- bandwidth: from $70 \mathrm{~Hz}$ to $100 \mathrm{kHz}(-3 \mathrm{~dB})$

- distortion: $<2.1 \%$ when input signal is between 14 and $28 \mathrm{mV}_{\mathrm{rms}}$ and $<1.8 \%$ when input signal is less than 14 $\mathrm{m} V_{\mathrm{rms}}$

3. Microphone preamplifier:

- input impedance. The measured input impedance amounts to $63 \mathrm{k} \Omega$. The difference between this value and our design aim of $50 \mathrm{k} \Omega$ is due to the relatively low 
value of the current gain factor $\beta_{F}$ of the vertical PNP transistors in the semicustom process, which is about 40 . This is also confirmed by simulations. Simulation of the input impedance of the microphone preamplifier using the model of the lateral PNP transistors that will be used in the fullcustom version $\left(\beta_{F} \approx 200\right)$ of the front-end yields an input impedance of $52 \mathrm{k} \Omega$, which is in good accordance with our design aim.

- noise. The input referred noise was calculated by measuring the spectral density of the noise current at the output of the front-end, dividing this spectrum by the measured transfer function of the front-end and multiplying the result by a polynome to obtain an A-weighted result. The input referred noise of the front-end amounts to 2.4 $\mu \mathrm{V}_{\mathrm{rms}}$. This is in accordance with simulations. When the input referred noise is simulated with lateral PNP transistors the result is $2.2 \mu \mathrm{V}_{\text {rms }}$, which is in good accordance with our design aim. As the rear-end of the hearing instrument operates in class-B, it is to be expected that its influence on the noise behavior of the total hearing instrument will not be noticeable.

- max. input signal:

a) $35 \mathrm{mV}_{\mathrm{rms}}$ unweighted for frequencies higher than $70 \mathrm{~Hz}$

b) $50 \mathrm{mV}_{\mathrm{rms}}$ unweighted for frequencies lower than $50 \mathrm{~Hz}$

4. Pickup-coil preamplifier:

- transfer: 5.4-55 k $\Omega$

- input impedance $(1 \mathrm{kHz}):<400 \Omega$

- output impedance $(1 \mathrm{kHz}): 4.0-4.7 \mathrm{k} \Omega$

- bandwidth: $>100 \mathrm{kHz}$

- output noise $(8 \mathrm{kHz}):<7.4 \mu \mathrm{V}_{\mathrm{rms}}$

- current consumption: $<21 \mu \mathrm{A}$

5. High-pass filter:

- order: 2 (real poles)

- cutoff frequency: $100 \mathrm{~Hz}-1.5 \mathrm{kHz}$ (linearly adjustable in octaves)

- stop-band attenuation: $>43 \mathrm{~dB}$

6. Low-pass filter:

- order: 2 (Butterworth characteristic)

- cutoff frequency: $1.5-7.8 \mathrm{kHz}$ (linearly adjustable in octaves)

- stop-band attenuation: $>27 \mathrm{~dB}$

7. AGC-I:

- attack time: $3.5 \mathrm{~ms}$

- release time: $55 \mathrm{~ms}$. The difference between this value and our design aim of $80 \mathrm{~ms}$ is again due to the relatively low value of the current gain factor $\beta_{F}$ of the vertical PNP transistors. This causes a large error in the inputoutput relation of the gm-compensated current mirror in the envelope processor, which, in turn, has a dominant effect on the release time. However, simulations of the front-end with lateral PNP transistors yields a release time of $81 \mathrm{~ms}$. Hence it is expected that the release time of the fullcustom version of the front-end will be in accordance with our design aim.
- knee level: $60 \mu \mathrm{V}_{\mathrm{rms}}-35 \mathrm{mV}_{\mathrm{rms}}$ (linearly adjustable in $\mathrm{dB}$ 's)

- compression ratio above knee level: 2

\section{Full Custom Realization of the Front-End}

All the circuits, of both the front-end and the rear-end, have been realized on a single chip in a $2.5-\mu \mathrm{m}$ BiCMOS process that contains vertical NPN's and PNP's as well as lateral PNP's. Because of contractual obligations no measurement results can be given here, except the general remark that the first measurements indicate a better performance than the semicustom version of the chip, due to the above mentioned reason of the higher current gain of the PNP transistors. The chip area of the front-end amounts to $2.4 \mathrm{~mm}^{2}$. For the total IC, including its bond pads, the chip area is about $5.1 \mathrm{~mm}^{2}$.

\section{CONCLUSION}

In this paper, the front-end of a universally applicable analog integrated circuit for hearing instruments has been presented, consisting of a microphone preamplifier, a pickup-coil preamplifier, a second-order high-pass filter and a second-order low-pass filter, both with a controllable cutoff frequency, and an input-controlled automatic gain control with an adjustable knee level. By applying a compression/expansion system and operating all the circuits in the current domain as much as possible, all these functions can be implemented in a single IC, without the need for external components. The semicustom test chip demonstrates operation down to $1.05 \mathrm{~V}$ and a current consumption between 120 and $175 \mu \mathrm{A}$. The full-custom chip area in a $2.5-\mu \mathrm{m}$ BiCMOS process (using only vertical NPN's and lateral PNP's) amounts to $2.4 \mathrm{~mm}^{2}$.

\section{REFERENCES}

[1] Market study, NSS (Dutch Foundation for Statistics), Jan. 1994.

[2] W. Soede, "Improvement of speech intelligibility in noise," Ph. D. dissertation, Delft University of Technology, Delft, The Netherlands, 1990.

[3] R. Plomp, "Acoustical aspects of cocktail parties," Acustica, vol. 38, pp. 186-191, 1977.

[4] M. R. Hutchinson, U.S. Patent No. 710,979, issued Oct. 1902.

[5] T. S. Littler, "Hearing aids for the deaf," J. Sci. Instr., vol. 13, pp. $144-155,1936$.

[6] E. Villchur, "Signal processing to improve speech intelligibility in perceptive deafness," J. Acoust. Soc. Am., vol. 53, pp. 1646-1657, June 1973.

[7] V. J. Geers, F. Keller, A. Löwe, and P. Plath, Technische Hilfe bei der Rehabilitation $H$ örgesch ädigter. Heidelberg, West Germany: Springer-Verlag, 1980.

[8] W. A. Serdijn, "The design of low-voltage low-power analog integrated circuits and their applications in hearing instruments," Delft University Press, Delft, 1994.

[9] — "A low-voltage low-power current-mode high-pass leapfrog filter," Analog Int. Circuits Signal Process., vol. 3, pp. 105-112, 1993.

[10] D. G. Haigh, J. T. Taylor, and B. Singh, "Continuous-time and switched capacitor monolithic filters based on current and charge simulation," Inst. Elect. Eng 'Proc., vol. 137, pt. G, pp. 147-154, Apr. 1990.

[11] W. A. Serdijn, A. C. van der Woerd, J. Davidse, and A. H. M. van Roermund," "Low-voltage low-power fully-integratable automatic gain controls," Analog Int. Circuits Signal Process., vol. 8, no. 2, pp. 121-133, 1995.

[12] W. A. Serdijn, A. C. van der Woerd, A. H. M. van Roermund, and J. Davidse, "Design principles for low-voltage low-power analog integrated circuits," Analog Int. Circuits Signal Process., vol. 8, no. 1, pp. $115-120,1995$.

[13] E. H. Nordholt, Design of high-performance negative-feedback amplifiers. Amsterdam, The Netherlands: Elsevier, 1983. 
[14] P. T. M. van Zeijl, "Fundamental aspects and design of an FM upconversion receiver front-end with on-chip SAW filters," Ph.D. dissertation, Delft Univ. Technol., Delft, The Netherlands.

[15] J. Fonderie and J. H. Huijsing, "1-V operational amplifier with multipath-driven output stage," IEEE J. Solid-State Circuits, vol. 26, Dec. 1991.

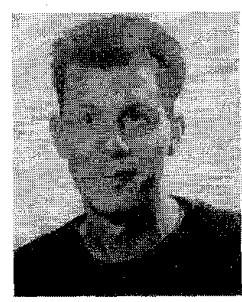

Wouter Serdijn was born in Zoetermeer, The Netherlands, in 1966. He started his course at the Faculty of Electrical Engineering at the Delft University of Technology in 1984, and received the "ingenieurs" (M.Sc.) degree in 1989. Subsequently, he joined the Electronics Research Laboratory of the same university where he received the Pl.D. degree in 1994

His research includes developing a formal design theory for low-voltage and ultra-low power analog integrated circuits along with the development of circuits for hearing instruments. He has edited two special issues on lowvoltage low-power analog integrated circuits for Kluwer's Analog Integrated Circuits and Signal Processing. He teaches electronic design techniques.

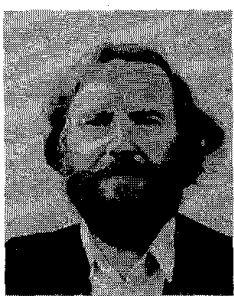

Albert C. van der Woerd was born in 1937 in Leiden, The Netherlands. In 1977, he received the "ingenieurs" (M.Sc.) degree in electrical engineering from the Delft University of Technology, Delft, The Netherlands. He was awarded the Ph. D. degree in 1985 .

From 1959 to 1966 , he was engaged in research on and development of radar and TV circuits at several industrial laboratories. In 1966, he joined the Electronics Research Laboratory of the Faculty of Electrical Engineering of the Delft University of Technology. During his first 11 years, he carried out research on electronic musical instruments. For the next eight years, his main research subject was carrier domain devices. More recently, he has specialized in the field of low-voltage low-power analog circuits and systems. He teaches desiga methodology.

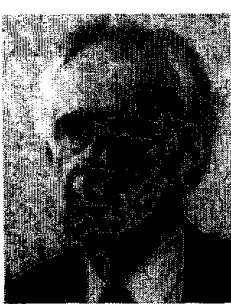

Jan Davidse received the M.Sc. degree in electrical engineering from the Delft University of Technology in 1953 and subsequently the Ph.D. degree from the Eindhoven University of Technology.

He was research scientist with Philips Research Laboratories and received a full professorship in electronics at the Delft University of Technology in 1964. From 1964 to 1994, he has been head of the Laboratory for Electronics at this University. His research interest is in analog electronics and in electronic pictorial techniques. He is the author of mumerous papers and holds several patents in his field of interest. He is the author of ten books, among which is Analog Electronic Circuit Design, (Prentice Hall, 1991).



Arthur H. M. van Roermund was born in Delft, The Netherlands in 1951. He received the M.Sc. degree in electrical engineering in 1975 from the Delft University of Technology and the Ph.D. degree in applied sciences from the K. U. Leuven, Belgium, in 1987.

From 1975 to 1992 he was with the Philips Research Laboratories in Eindhoven. First he joined the Consumer Electronics Group, where he has been involved with the design and integration of analog circuits and systems, especially switched-capacitor circuits. In 1987 he joined the Visual Communications Group where he has been engaged in video architectures and digital video signal processing. From 1987 to 1990 he was project leader of the Video Signal Processor project and from 1990 to 1992 the leader of a Multi-Window Television project. Since 1992 he has been a full professor with the Faculty of Electrical Engineering. Delft University of Technology. He heads the Electronics Laboratory, which is part of DIMES: the Delft Institute of Micro Electronics and Submicron Technology. 